\title{
On Discrete Solutions for Pseudo-Differential Equations
}

\author{
Vladimir Vasilyev ${ }^{1, a)}$ \\ ${ }^{1}$ Belgorod National Research University, Studencheskaya 14/1, Belgorod 308007, Russia. \\ a) Corresponding author: vladimir.b.vasilyev@ gmail.com
}

\begin{abstract}
We study model discrete pseudo-differential equations in some canonical domains of Euclidean space. We need such consideration to obtain approximate solution for initial continuous pseudo-differential equations and related boundary value problems. We call solutions of these discrete equations by discrete solutions for pseudo-differential equations. Our main goal is finding discrete solutions and comparison between discrete and continuous solutions.
\end{abstract}

\section{INTRODUCTION}

A certain theory of pseudo-differential operators and corresponding equations was constructed in the second half of the last century $[1,2,3]$, and it includes as usual boundedness theorems in different functional spaces and a certain variant of symbolic calculus. But for discrete situation there is no any variant of such a theory although there are a lot of approximate constructions for solving simplest kinds of pseudo-differential equations, for example singular integral and similar equations $[4,5,6]$. Moreover there are some recent studies for these discrete situations from algebraic or symbolic calculus point of view on the whole $m$-dimensional lattice $\mathbb{Z}^{m}$. But there are principal difficulties to transfer this approach to another discrete domains which are not $\mathbb{Z}^{m}$, for example a discrete half-space or a discrete cone.

\section{DISCRETE SPACES AND OPERATORS}

For studying discrete analogues of pseudo-differential equations $[1,2,3]$ we will use the discrete Fourier transform for defining discrete Sobolev-Slobodetskii spaces; such discrete spaces are very convenient in the theory.

Let us denote by $u_{d}(\tilde{x})$ a function of a discrete variable $\tilde{x} \in h \mathbb{Z}^{m}, h>0$, and by $\tilde{u}_{d}(\xi), \xi \in \hbar \mathbb{T}^{m}, \hbar=h^{-1}, \mathbb{T}^{m}=$ $[-\pi, \pi]^{m}$, its discrete Fourier transform

$$
\left(F_{d} u_{d}\right)(\xi)=\sum_{\tilde{x} \in h \mathbb{Z}^{m}} u_{d}(\tilde{x}) e^{i \tilde{x} \xi} h^{m}
$$

We denote $\zeta^{2}=h^{-2} \sum_{k=1}^{m}\left(e^{-i h \xi_{k}}-1\right)^{2}$ and introduce the following

Definition 1. The space $H^{s}\left(h \mathbb{Z}^{m}\right)$ of discrete functions $u_{d}(\tilde{x})$ with the finite norm

$$
\left\|u_{d}\right\|_{s}=\left(\int_{h T^{m}}\left(1+\left|\zeta^{2}\right|\right)^{s}\left|\tilde{u}_{d}(\xi)\right|^{2} d \xi\right)^{1 / 2}
$$
domain.

Further, we introduce $D=\mathbb{R}_{+}^{m}=\left\{x \in \mathbb{R}^{m}: x=\left(x^{\prime}, x_{m}\right), x_{m}>0\right\}$, and $D_{d}=D \cap h \mathbb{Z}^{m}$ is a corresponding discrete

Definition 2. The space $H^{s}\left(D_{d}\right)$ consists of discrete functions from $H^{s}\left(h \mathbb{Z}^{m}\right)$ with supports in $\overline{D_{d}}$. A norm in the space $H^{s}\left(D_{d}\right)$ is induced by a norm of the space $H^{s}\left(h \mathbb{Z}^{m}\right)$. The space $H_{0}^{s}\left(D_{d}\right)$ consists of discrete functions $u_{d}$ with supports in $D_{d}$ and these functions must admit a continuation $\ell u_{d}$ on the whole space $H^{s}\left(h \mathbb{Z}^{m}\right)$. A norm in the space $H_{0}^{s}\left(D_{d}\right)$ is given by the formula

$$
\left\|u_{d}\right\|_{s}^{+}=\inf \left\|\ell u_{d}\right\|_{s}
$$

International Conference of Numerical Analysis and Applied Mathematics (ICNAAM 2018)

AIP Conf Proc. 2116, 040010-1-040010-4; https//doi org/10 1063/15114031

Published by AIP Publishing 978-0-7354-1854-7/ $\$ 3000$

040010-1 
where infimum is taken over all continuations $\ell$.

Let $A_{d}(\xi)$ be a periodic function in $\mathbb{R}^{m}$ with basic cube of periods $\hbar \mathbb{T}^{m}$. Such functions we call symbols.

Definition 3. A digital pseudo-differential operator $A_{d}$ in the discrete domain $D_{d}$ is called an operator of the following type

$$
\left(A_{d} u_{d}\right)(\tilde{x})=\sum_{\tilde{y} \in h \mathbb{Z}^{m}} \int_{\hbar \pi^{m}} A_{d}(\xi) e^{i(\tilde{x}-\tilde{y}) \cdot \xi} \tilde{u}_{d}(\xi) d \xi, \quad \tilde{x} \in D_{d},
$$

We will consider class $E_{\alpha}$ of symbols satisfying the following condition

$$
c_{1}\left(1+\left|\zeta^{2}\right|\right)^{\alpha / 2} \leq\left|A_{d}(\xi)\right| \leq c_{2}\left(1+\left|\zeta^{2}\right|\right)^{\alpha / 2}
$$

with constants $c_{1}, c_{2}$ non-depending on $h$.

Let us denote $\Pi_{ \pm}=\left\{\left(\xi^{\prime}, \xi_{m} \pm i \tau\right), \tau>0\right\}, \xi=\left(\xi^{\prime}, \xi_{m}\right) \in \mathbb{T}^{m}$.

\section{SOLVABILITY}

We are interested in a solvability of the equation

$$
\left(A_{d} u_{d}\right)(\tilde{x})=v_{d}(\tilde{x}), \quad \tilde{x} \in D_{d}
$$

in the space $H^{s}\left(D_{d}\right)$ if the right hand side $v_{d} \in H_{0}^{s-\alpha}\left(D_{d}\right)$.

This discrete equation (1) is a discrete analogue of certain continuous equation

$$
(A u)(x)=v(x), \quad x \in D
$$

with a pseudo-differential operator $A$ with the symbol $A(\xi)$.

To obtain solvability results on the discrete equation (1) we use the following concept $[3,7,8,9,10]$.

Definition 4. A periodic factorization of an elliptic symbol $A_{d}(\xi) \in E_{\alpha}$ is called its representation in the form

$$
A_{d}(\xi)=A_{d,+}(\xi) A_{d,-}(\xi),
$$

where the factors $A_{d, \pm}(\xi)$ admit an analytic continuation into half-strips $\hbar \Pi_{ \pm}$on the last variable $\xi_{m}$ for almost all fixed $\xi^{\prime} \in \hbar \mathbb{T}^{m-1}$ and satisfy the estimates

$$
\left|A_{d,+}^{ \pm 1}(\xi)\right| \leq c_{1}\left(1+\left|\hat{\zeta}^{2}\right|\right)^{ \pm \frac{x}{2}}, \quad\left|A_{d,-}^{ \pm 1}(\xi)\right| \leq c_{2}\left(1+\left|\hat{\zeta}^{2}\right|\right)^{ \pm \frac{\alpha-x}{2}},
$$

with constants $c_{1}, c_{2}$ non-depending on $h$,

$$
\hat{\zeta}^{2} \equiv \hbar^{2}\left(\sum_{k=1}^{m-1}\left(e^{-i \hbar \xi_{k}}-1\right)^{2}+\left(e^{-i \hbar\left(\xi_{m}+i \tau\right)}-1\right)^{2}\right), \quad \xi_{m}+i \tau \in \hbar \Pi_{ \pm} .
$$

The number $\mathfrak{x} \in \mathbb{R}$ is called an index of periodic factorization.

Remark 1. Such periodic factorization exists at least for smooth symbols $A_{d}(\xi)$.

Let us denote by $\ell v_{d}$ an arbitrary continuation of $v_{d}$ into a whole $h \mathbb{Z}^{m}$.

Theorem 1. If the elliptic symbol $A_{d}(\xi) \in E_{\alpha}$ admits periodic factorization with index $\mathfrak{x}$ so that $|\mathfrak{x}-s|<1 / 2$ then the the equation (1) has unique solution in the space $H^{s}\left(D_{d}\right)$

$$
\begin{gathered}
\tilde{u}_{d}(\xi)=A_{d,+}^{-1}(\xi) P_{\xi^{\prime}}^{\text {per }}\left(A_{d,-}^{-1}(\xi) \widetilde{\ell v_{d}}(\xi)\right) . \\
\left(P_{\xi^{\prime}}^{\text {per }} \tilde{u}_{d}\right)(\xi) \equiv \frac{1}{2}\left(\tilde{u}_{d}(\xi)+\frac{h}{2 \pi i} v \cdot p \cdot \int_{-\hbar \pi}^{\hbar \pi} \tilde{u}_{d}\left(\xi^{\prime}, \eta_{m}\right) \cot \frac{h\left(\xi_{m}-\eta_{m}\right)}{2} d \eta_{m}\right),
\end{gathered}
$$

for arbitrary right-hand side $v_{d} \in H_{0}^{s-\alpha}\left(D_{d}\right)$, 
Theorem 2. Let $\mathfrak{x}-s=n+\delta, n \in \mathbf{N},|\delta|<1 / 2$. Then a general solution of the equation (1) in Fourier images has the following form

$$
\tilde{u}_{d}(\xi)=A_{d,+}^{-1}(\xi) X_{n}(\xi) P_{\xi^{\prime}}^{p e r}\left(X_{n}^{-1}(\xi) A_{d,-}^{-1}(\xi) \widetilde{\ell v_{d}}(\xi)\right)+A_{d,+}^{-1}(\xi) \sum_{k=0}^{n-1} c_{k}\left(\xi^{\prime}\right) \hat{\zeta}_{m}^{k},
$$

where $X_{n}(\xi)$ is an arbitrary polynomial of order $n$ of variables $\hat{\zeta}_{k}=\hbar\left(e^{-i \hbar \xi_{k}}-1\right), k=1, \cdots, m$, satisfying the condition (2), $c_{k}\left(\xi^{\prime}\right), j=0,1, \cdots, n-1$, are arbitrary functions from $H_{s_{k}}\left(h \mathbf{T}^{m-1}\right), s_{k}=s-\mathfrak{x}+k-1 / 2$.

Theorem 3. Let $\mathfrak{x}-s=-n+\delta, n \in \mathbb{N},|\delta|<1 / 2$. Then the equation (1) has a solution in the space $H^{s}\left(D_{d}\right)$ iff

$$
c_{j}\left(\xi^{\prime}\right)=0, \forall a . a . \xi^{\prime} \in \hbar \mathbb{T}^{m-1}, j=0,1, \cdots, n .
$$

where

$$
c_{j}\left(\xi^{\prime}\right)=\int_{-\hbar \pi}^{\hbar \pi}\left(e^{i h \xi_{m}}-1\right)^{j} A_{d,-}^{-1}\left(\xi^{\prime}, \xi_{m}\right)\left(\widetilde{\ell v_{d}}\right)\left(\xi^{\prime}, \xi_{m}\right) d \xi_{m}, \quad j=0,1, \cdots, n,
$$

Remark 2. One can easily verify that all assertions of theorems $1-3$ do not depend on the continuation $\ell v_{d}$.

\section{COMPARISON}

Let $A(\xi)$ be a locally integrable function in $\mathbb{R}^{m}$ satisfying the condition

$$
c_{1}(1+|\xi|)^{\alpha} \leq|A(\xi)| \leq c_{2}(1+|\xi|)^{\alpha},
$$

where $c_{1}, c_{2}$ are constants. We will consider the equation (2) where the operator $A$ has such symbol $A(\xi)$. Then we construct the periodic symbol $A_{d}(\xi)$ in the following way. We take a restriction of $A(\xi)$ on the cube $\hbar T^{m}$ and periodically extend it onto a whole $\mathbb{R}^{m}$. We consider such discrete operator $A_{d}$ as an approximate operator for $A$.

A construction for the restriction operator $Q_{h}$ for functions $u \in S\left(\mathbb{R}^{m}\right)$ is the following. We take the Fourier transform $\tilde{u}(\xi)$, then its restriction on $\hbar \mathbb{T}^{m}$ and periodically continue it onto a whole $\mathbb{R}^{m}$. Further we apply the inverse discrete Fourier transform

$$
\left(F_{d}^{-1} \tilde{u}_{d}\right)(\tilde{x})=\frac{1}{(2 \pi)^{m}} \int_{\hbar T^{m}} e^{-i \tilde{x} \xi} \tilde{u}_{d}(\xi) d \xi, \quad \tilde{x} \in h \mathbb{Z}^{m}
$$

and obtain a discrete function which is denoted by $\left(Q_{h} u\right)(\tilde{x}), \tilde{x} \in h \mathbb{Z}^{m}$.

Further, we introduce under consideration the following discrete equation

$$
\left(A_{d} u_{d}\right)(\tilde{x})=\left(Q_{h}(\ell v)\right)(\tilde{x}), \quad \tilde{x} \in D_{d},
$$

Definition 5. A discrete solution for the equation (2) is called a solution of the equation (3) if it exists.

Let $S\left(\mathbb{R}^{m}\right)$ be the Schwartz class of infinitely differentiable rapidly decreasing at infinity functions; this class is dense in every space $H^{s}\left(\mathbb{R}^{m}\right)$ [3]. If we will put some conditions on the symbol $A(\xi)$ then we can compare discrete and continuous solutions. Moreover, we suppose that $|\mathfrak{X}-s|<1 / 2$ and the equation (2) is uniquely solvable in the space $H^{s}(D)$ for an arbitrary right hand side $v \in H_{0}^{s-\alpha}(D)$, and $A_{ \pm}(\xi)$ are elements of factorization in Eskin's sense [3]. Then we have the following result.

Theorem 4. If the symbol $A(\xi)$ satisfies the condition and is infinitely differentiable in $\mathbb{R}^{m}$ with the factors $A_{ \pm}(\xi)$, $u$ is a solution of the equation (2), $u_{d}$ is a solution of the equation (3) then for $v \in S\left(\mathbb{R}^{m}\right)$ we have the following error estimate

$$
\left|u(\tilde{x})-u_{d}(\tilde{x})\right| \leq C h^{\beta}, \quad \forall \tilde{x} \in D_{d},
$$

for arbitrary $\beta>0$.

\section{CONCLUSION}

Of course these studies are initial results in this direction. More complicated situations related to boundary value problems [9] as well to a conical singularity $[11,12]$ require separate studying. 


\section{ACKNOWLEDGMENTS}

This work was supported by the State contract of the Russian Ministry of Education and Science (contract No 1.7311.2017/8.9).

\section{REFERENCES}

[1] M. Taylor, Pseudodifferential Operators (Princeton University Press, Princeton, 1981).

[2] F. Treves, Introduction to Pseudodifferential Operators and Fourier Integral Operators (Springer, New York, 1980).

[3] G. Eskin, Boundary Value Problems for Elliptic Pseudodifferential Equations (AMS, Providence, 1981).

[4] A. V. Vasil'ev and V. B. Vasil'ev, Differ. Equ. 51, 652-660 (2015).

[5] A. Vasil'ev and V. Vasil'ev, Doklady Math. 92, 585-589 (2015).

[6] A. Vasilyev and V. Vasilyev, "Discrete singular integrals in a half-space," in Current Trends in Analysis and Its Applications, Trends in Mathematics. Research Perspectives, edited by V. Mityushev and M. Ruzhansky (Birkhauser, Basel, 2015), pp. 663-670.

[7] A. V. Vasil'ev and V. B. Vasil'ev, Math. Model. Anal. 23, 492-506 (2018).

[8] V. B. Vasilyev, "Discreteness, periodicity, holomorphy, and factorization," in Integral Methods in Science and Engineering. V.1. Theoretical Technique, edited by C. Constanda, M. D. Riva, P. Lamberti, and P. Musolino (Birkhauser/Springer, Cham, 2017), pp. 315-324.

[9] V. Vasilyev, "On discrete boundary value problems," in International Conference Functional Analysis in Interdisciplinary Applications (FAIA2017), AIP Conf. Proc., Vol. 1880, edited by T. Kal'menov and M. Sadybekov (AIP Publishing, Melville, 2017) p. 050010.

[10] A. Vasilyev and V. Vasilyev, "On some discrete boundary value problems in canonical domains," in Differential and Difference Equations with Applications. ICDDEA, Amadora, Portugal, June 2017, Springer Proceedings in Mathematics \& Statistics, Vol. 230, edited by S. Penelas, T. Caraballo, P. Kloeden, and J. Graef (Birkhauser/Springer, Cham, 2018), pp. 569-579.

[11] V. B. Vasil'ev, Wave Factorization of Elliptic Symbols: Theory andApplications. Introduction to the Theory of Boundary Value Problems in Non-Smooth Domains (Kluwer Academic Publishers, Dordrecht-BostonLondon, 2000).

[12] V. Vasilyev, "The periodic cauchy kernel, the periodic bochner kernel, discrete pseudo-differential operators," in Proceedings of the International Conference on Numerical Analysis and Applications (ICNAAM-2016), AIP Conf. Proc., Vol. 1863, edited by T. Simos and C. Tsitouras (AIP Publishing, Melville, 2017) p. 140014. 